\title{
Improved Exponential Type Estimators for Finite Population Mean in Stratified Random Sampling
}

\author{
Nursel KOYUNCU \\ Hacettepe University, Department of Statistics, Beytepe, Ankara, Turkey \\ nkoyuncu@ hacettepe.edu.tr
}

\begin{abstract}
In this paper new exponential type estimators are suggested for estimating the population mean in stratified random sampling. The biases and mean square errors of the suggested estimators are obtained. Also an empirical study is carried out to show the properties of the proposed estimators.
\end{abstract}

Keywords: Ratio estimator; Exponential estimator; Mean square error; Efficiency; Stratified random sampling.

\section{Introduction}

In sampling theory the role of auxiliary information has a great importance. It is well known that using suitable auxiliary information such as population total, mean, skewness, correlation, attribute etc. can improve precision of estimates. Many authors have used this information in their ratio, product and exponential type estimators to get more efficient estimator under different sampling design. In stratified random sampling scheme the authors including Diana (1993), Singh and Vishwakarma (2008), Koyuncu and Kadilar (2009, 2010), Tailor et al. (2012), Koyuncu (2013), Yadav et al. (2014) etc. have suggested estimators using auxiliary information. In this paper we have tried to generalize Singh et al. (2008) and Yadav et al. (2014) estimators and suggested two exponential type estimators in stratified random sampling.

Consider a finite population $U=\left(u_{1}, u_{2}, \ldots, u_{N}\right)$ of size $N$ and let $y$ and $x$, respectively, be the study and auxiliary variable associated with each unit $u_{j}(j=1,2, \ldots ., N)$ of the population. Assume that the population of size, $N$, is stratified into $L$ strata with $h$-th stratum containing $N_{h}$ units, where $h=1,2, \ldots, L$ such that $\sum_{h=1}^{L} N_{h}=N$. A simple random sample of size $n_{h}$ is drawn without replacement from the $h$-th stratum such that $\sum_{h=1}^{L} n_{h}=n$. Let $\left(y_{h i}, x_{h i}\right)$ denote the observed values of $y$ and $x$ on the $i$-th unit of the $h$-th stratum, where $i=1,2, \ldots, N_{h}$. Moreover, let $\bar{y}_{h}=\sum_{i=1}^{n_{h}} \frac{y_{h i}}{n_{h}}, \bar{y}_{s t}=\sum_{h=1}^{L} W_{h} \bar{y}_{h}$, and $\bar{Y}_{h}=\sum_{i=1}^{N_{h}} \frac{y_{h i}}{N_{h}}$, $\bar{Y}=\bar{Y}_{s t}=\sum_{h=1}^{L} W_{h} \bar{Y}_{h}$ be the sample and population means of $y$, respectively, where $W_{h}=\frac{N_{h}}{N}$ is the stratum weight. 
To obtain the bias and MSE, let us define $e_{0}=\left(\bar{y}_{s t}-\bar{Y}\right) / \bar{Y}$ and $e_{1}=\left(\bar{x}_{s t}-\bar{X}\right) / \bar{X}$. Using these notations, $E\left(e_{0}\right)=E\left(e_{1}\right)=0$,

$$
V_{r s}=\sum_{h=1}^{L} W_{h}^{r+s} \frac{E\left\lfloor\left(\bar{y}_{h}-\bar{Y}_{h}\right)^{r}\left(\bar{x}_{h}-\bar{X}_{h}\right)^{s}\right\rfloor}{\bar{Y}^{r} \bar{X}^{s}}
$$

Using (1), we can write

$$
E\left(e_{0}^{2}\right)=\frac{\sum_{h=1}^{L} W_{h}^{2} \lambda_{h} S_{y h}^{2}}{\bar{Y}^{2}}=V_{20}, E\left(e_{1}^{2}\right)=\frac{\sum_{h=1}^{L} W_{h}^{2} \lambda_{h} S_{x h}^{2}}{\bar{X}^{2}}=V_{02} \quad E\left(e_{0} e_{1}\right)=\frac{\sum_{h=1}^{L} W_{h}^{2} \lambda_{h} S_{x y h}}{\bar{X} \bar{Y}}=V_{11}
$$

where

$$
S_{y h}^{2}=\frac{\sum_{i=1}^{N_{h}}\left(y_{h i}-\bar{Y}_{h}\right)^{2}}{N_{h}-1}, \quad S_{x h}^{2}=\frac{\sum_{i=1}^{N_{h}}\left(x_{h i}-\bar{X}_{h}\right)^{2}}{N_{h}-1}, S_{x y h}=\frac{\sum_{i=1}^{N_{h}}\left(x_{h i}-\bar{X}_{h}\right)\left(y_{h i}-\bar{Y}_{h}\right)}{N_{h}-1}, \lambda_{h}=\frac{1-f_{h}}{n_{h}},
$$

and $f_{h}=\frac{n_{h}}{N_{h}}$.

Singh et al. (2008) have suggested estimators of population mean using auxiliary information, given by

$$
\begin{aligned}
& t_{1}=\bar{y}_{s t} \exp \left(\frac{\bar{X}_{1}-\bar{x}_{1}}{\bar{X}_{1}+\bar{x}_{1}}\right) \\
& t_{2}=\bar{y}_{s t} \exp \left(\frac{\bar{X}_{2}-\bar{x}_{2}}{\bar{X}_{2}+\bar{x}_{2}}\right)
\end{aligned}
$$

where $\quad \bar{x}_{1}=\sum_{h=1}^{L} W_{h}\left(\bar{x}_{h}+C_{x h}\right), \quad \bar{X}_{1}=\sum_{h=1}^{L} W_{h}\left(\bar{X}_{h}+C_{x h}\right), \quad \bar{x}_{2}=\sum_{h=1}^{L} W_{h}\left(\bar{x}_{h}+\beta_{2 h}(x)\right) \quad$ and $\bar{X}_{2}=\sum_{h=1}^{L} W_{h}\left(\bar{X}_{h}+\beta_{2 h}(x)\right)$.

To generalize $t_{1}$ and $t_{2}$ estimators in a class we can define

$$
k_{1}=\bar{y}_{s t} \exp \left(\frac{\bar{X}-\bar{x}_{s t}}{\bar{X}+\bar{x}_{s t}+2 A_{s t}}\right)
$$

where $A_{s t}=\sum_{h=1}^{L} W_{h} A_{h}$ and $A_{h}$ may be some population information of the auxiliary variable for $h$-th stratum such as $S_{x h}$, coefficient of variation $C_{x h}$, skewness $\beta_{1 h}(x)$, kurtosis $\beta_{2 h}(x)$, correlation coefficient $\rho_{h(x y)}$. Note that $t_{1}$ and $t_{2}$ estimators are member of $k_{1}$ if we take $A_{h}$ as $C_{x h}$ and $\beta_{2 h}(x)$ respectively as given in Table2.

Expressing (4) in terms of e's, we have

$$
\left(k_{1}-\bar{Y}\right)=\bar{Y}\left\{-\frac{\bar{X}}{2\left(\bar{X}+A_{s t}\right)} e_{1}+\frac{3 \bar{X}^{2}}{8\left(\bar{X}+A_{s t}\right)^{2}} e_{1}^{2}+e_{0}-\frac{\bar{X}}{2\left(\bar{X}+A_{s t}\right)} e_{0} e_{1}\right\}
$$


Squaring both sides of (5) and neglecting the terms of e's having greater than two, we can write

$$
\left(k_{1}-\bar{Y}\right)^{2}=\bar{Y}^{2}\left\{e_{0}^{2}+\frac{\bar{X}^{2}}{4\left(\bar{X}+A_{s t}\right)^{2}} e_{1}^{2}-\frac{\bar{X}}{\left(\bar{X}+A_{s t}\right)} e_{0} e_{1}\right\}
$$

Taking expectation of (6) we get the MSE of $k_{1}$, under the first order of approximation is given by

$$
\begin{aligned}
\operatorname{MSE}\left(k_{1}\right) & =\left\{\sum_{h=1}^{L} W_{h}^{2} \lambda_{h} S_{y h}^{2}+\frac{\bar{Y}^{2}}{4\left(\bar{X}+A_{s t}\right)^{2}} \sum_{h=1}^{L} W_{h}^{2} \lambda_{h} S_{x h}^{2}-\frac{\bar{Y}}{\left(\bar{X}+A_{s t}\right)} \sum_{h=1}^{L} W_{h}^{2} \lambda_{h} S_{x y h}\right\} \\
& =\sum_{h=1}^{L} W_{h}^{2} \lambda_{h}\left(S_{y h}^{2}+\frac{\bar{Y}}{4\left(\bar{X}+A_{s t}\right)^{2}} S_{x h}^{2}-\frac{\bar{Y}}{\left(\bar{X}+A_{s t}\right)} S_{x y h}\right) \\
& =\sum_{h=1}^{L} W_{h}^{2} \lambda_{h}\left(S_{y h}^{2}+\frac{1}{4} R_{k 1}^{2} S_{x h}^{2}-R_{k 1} S_{x y h}\right)
\end{aligned}
$$

where $R_{k 1}=\frac{\bar{Y}}{\left(\bar{X}+A_{s t}\right)}$.

Secondly Singh et al. (2008) have suggested estimators using more auxiliary information

$$
\begin{aligned}
& t_{3}=\bar{y}_{s t} \exp \left(\frac{\bar{X}_{3}-\bar{x}_{3}}{\bar{X}_{3}+\bar{x}_{3}}\right) \\
& t_{4}=\bar{y}_{s t} \exp \left(\frac{\bar{X}_{4}-\bar{x}_{4}}{\bar{X}_{4}+\bar{x}_{4}}\right)
\end{aligned}
$$

where $\bar{x}_{3}=\sum_{h=1}^{L} W_{h}\left(\bar{x}_{h} \beta_{2 h}(x)+C_{x h}\right), \quad \bar{X}_{3}=\sum_{h=1}^{L} W_{h}\left(\bar{X}_{h} \beta_{2 h}(x)+C_{x h}\right)$,

$$
\bar{x}_{4}=\sum_{h=1}^{L} W_{h}\left(\bar{x}_{h} C_{x h}+\beta_{2 h}(x)\right), \quad \bar{X}_{4}=\sum_{h=1}^{L} W_{h}\left(\bar{X}_{h} C_{x h}+\beta_{2 h}(x)\right)
$$

To generalize $t_{3}$ and $t_{4}$ estimators let us define following class

$$
k_{2}=\bar{y}_{s t} \exp \left(\frac{A_{s t}^{*}-a_{s t}^{*}}{A_{s t}^{*}+a_{s t}^{*}+2 B_{s t}}\right)
$$

where $A_{s t}^{*}=\sum_{h=1}^{L} W_{h} \bar{X}_{h} A_{h}, a_{s t}^{*}=\sum_{h=1}^{L} W_{h} \bar{x}_{h} A_{h}, B_{s t}=\sum_{h=1}^{L} W_{h} B_{h}, A_{h}$ and $B_{h}$ may be some population information of the auxiliary variable for $h$-th stratum such as $S_{x h}$, coefficient of variation $C_{x h}$, skewness $\beta_{1 h}(x)$, kurtosis $\beta_{2 h}(x)$, correlation coefficient $\rho_{h(x y)}$.

To obtain the bias and MSE, let us define

$$
e_{1}^{*}=\frac{\left(a_{s t}^{*}-A_{s t}^{*}\right)}{A_{s t}^{*}}=\frac{\sum_{h=1}^{L} W_{h} A_{h}\left(\bar{x}_{h}-\bar{X}_{h}\right)}{A_{s t}^{*}}, \quad V_{r, s, t}^{*}=\sum_{h=1}^{L} W_{h}^{r+s} A_{h}^{s} \frac{E\left[\left(\bar{y}_{h}-\bar{Y}_{h}\right)^{r}\left(\bar{x}_{h}-\bar{X}_{h}\right)^{s+t}\right]}{\bar{Y}^{r} A_{s t}^{* s} \bar{X}^{t}}
$$




\section{Nursel KOYUNCU}

Using these notations we can write following notations

$$
\begin{aligned}
& E\left(e_{0}^{2}\right)=V_{200}^{*}=V_{20} \quad E\left(e_{1}^{* 2}\right)=\frac{\sum_{h=1}^{L} W_{h}^{2} A_{h}^{2} \lambda_{h} S_{x h}^{2}}{A_{s t}^{* 2}}=V_{020}^{*} \\
& E\left(e_{0} e_{1}^{*}\right)=\frac{\sum_{h=1}^{L} W_{h}^{2} A_{h} \lambda_{h} S_{x y h}}{\bar{Y} A_{s t}^{*}}=V_{110}^{*} \\
& E\left(e_{1} e_{1}^{*}\right)=\frac{\sum_{h=1}^{L} W_{h}^{2} A_{h} \lambda_{h} S_{x}^{2}}{\bar{X} A_{s t}^{*}}=V_{011}^{*}, \quad E\left(e_{1}^{2}\right)=\frac{\sum_{h=1}^{L} W_{h}^{2} \lambda_{h} S_{x h}^{2}}{\bar{X}^{2}}=V_{002}^{*}=V_{02}, \\
& E\left(e_{0} e_{1}\right)=\frac{\sum_{h=1}^{L} W_{h}^{2} \lambda_{h} S_{x y h}}{\bar{X} \bar{Y}}=V_{101}^{*}=V_{11} .
\end{aligned}
$$

Expressing (10) in terms of e's, we have

$$
k_{2}-\bar{Y}=\bar{Y}\left(-\frac{A_{s t}^{*}}{2\left(A_{s t}^{*}+B_{s t}\right)} e_{1}^{*}+\frac{3}{8} \frac{\left(A_{s t}^{*}\right)^{2}}{\left(A_{s t}^{*}+B_{s t}\right)^{2}} e_{1}^{* 2}+e_{0}-\frac{1}{2} A_{s t}^{*} e_{0} e_{1}^{*}+\frac{3}{8} \frac{\left(A_{s t}^{*}\right)^{2}}{\left(A_{s t}^{*}+B_{s t}\right)^{2}} e_{0} e_{1}^{* 2}\right)
$$

Squaring both sides of (11) and neglecting the terms of e's having greater than two, we can write

$$
\left(k_{2}-\bar{Y}\right)^{2}=\bar{Y}^{2}\left(e_{0}^{2}+\frac{1}{4\left(A_{s t}^{*}+C_{s t}\right)^{2}} A_{s t}^{* 2} e_{1}^{* 2}-\frac{A_{s t}^{*}}{\left(A_{s t}^{*}+C_{s t}\right)} e_{1}^{*} e_{0}\right)
$$

Taking expectation both sides of (12) then we get corrected MSE of Singh et al. (2008) as follows:

$$
\begin{aligned}
\operatorname{MSE}\left(k_{2}\right) & =\bar{Y}^{2}\left(\frac{\sum_{h=1}^{L} W_{h}^{2} \lambda_{h} S_{y h}^{2}}{\bar{Y}^{2}}+\frac{A_{s t}^{* 2}}{4\left(A_{s t}^{*}+C_{s t}\right)^{2}} \frac{\sum_{h=1}^{L} W_{h}^{2} A_{h}^{2} \lambda_{h} S_{x h}^{2}}{A_{s t}^{* 2}}-\frac{A_{s t}^{*}}{\left(A_{s t}^{*}+C_{s t}\right)} \frac{\sum_{h=1}^{L} W_{h}^{2} A_{h} \lambda_{h} S_{x y h}}{\bar{Y} A_{s t}^{*}}\right) \\
& =\sum_{h=1}^{L} W_{h}^{2} \lambda_{h}\left\{S_{y h}^{2}-\frac{\bar{Y}}{\left(A_{s t}^{*}+C_{s t}\right)} A_{h} S_{x y h}+\frac{1}{4} \frac{\bar{Y}^{2}}{\left(A_{s t}^{*}+C_{s t}\right)^{2}} A_{h}^{2} S_{x h}^{2}\right\} \\
& =\sum_{h=1}^{L} W_{h}^{2} \lambda_{h}\left\{S_{y h}^{2}-R_{k 2} S_{x y h}+\frac{1}{4} R_{k 2}^{2} S_{x h}^{2}\right\}
\end{aligned}
$$

where $R_{k 2}=\frac{\bar{Y} A_{h}}{\left(A_{s t}^{*}+B_{s t}\right)}$. 
Note that $t_{3}$ and $t_{4}$ estimators are member of $k_{2}$ for appropriate values of $A_{h}$ and $B_{h}$ as given in Table2. Also we have corrected the MSE of $t_{3}$ and $t_{4}$ estimators as given in Appendix with detail.

Yadav et al. (2014) have suggested ratio and product exponential estimators are given by

$$
\begin{gathered}
t_{R C}^{(a)}=\bar{y}_{s t} \exp \left(\frac{\bar{X}-\bar{x}_{s t}}{\bar{X}+(a-1) \bar{x}_{s t}}\right) \\
t_{P C}^{(b)}=\bar{y}_{s t} \exp \left(\frac{\bar{x}_{s t}-\bar{X}}{\bar{X}+(b-1) \bar{x}_{s t}}\right) \\
M S E_{\min }\left(t_{P C}^{(b)}\right)=M S E_{\min }\left(t_{R C}^{(a)}\right)=\bar{Y}^{2} V_{20}\left[1-\frac{V_{11}^{2}}{V_{02} V_{20}}\right]=\sum_{h=1}^{L} W_{h}^{2} \lambda_{h} S_{y h}^{2}\left\{1-\rho_{c}^{2}\right\} \\
\text { where } \rho_{c}=\frac{\sum_{h=1}^{L} W_{h}^{2} \lambda_{h} S_{x y h}}{\sqrt{\sum_{h=1}^{L} W_{h}^{2} \lambda_{h} S_{x h}^{2}} \sqrt{\sum_{h=1}^{L} W_{h}^{2} \lambda_{h} S_{y h}^{2}}} .
\end{gathered}
$$

In sampling literature many authors have suggested estimators using auxiliary information. Koyuncu and Kadilar (2010) defined a general combined class in stratified random sampling to get a compact expression for the asymptotic variance and avoid tedious calculations, given by

$$
t_{c}=g\left(\bar{y}_{s t}, u_{s t}\right)
$$

where $u_{s t}=\bar{x}_{s t} / \bar{X}$ and $g\left(\bar{y}_{s t}, u_{s t}\right)$ is a function of $\bar{y}_{s t}$ and $u_{s t}$. To study the properties of $t_{c}$ we assume following regularity conditions:

1. The point $\left(\bar{y}_{s t}, u_{s t}\right)$ assumes the value in a closed convex subset $R_{2}$ of two dimensional real space containing the point $(\bar{Y}, 1)$,

2. The function $g\left(\bar{y}_{s t}, u_{s t}\right)$ is continuous and bounded in $R_{2}$,

3. $g(\bar{Y}, 1)=\bar{Y}$ and $g_{0}(\bar{Y}, 1)=1$, where $g_{0}(\bar{Y}, 1)$ denotes the first order partial derivative of $g$ with respect to $\bar{y}_{s t}$,

4. The first and second order partial derivatives of $g\left(\bar{y}_{s t}, u_{s t}\right)$ exist and are continuous and bounded in $R_{2}$.

Note that this class contains many estimator. We can say that Yadav et al. (2014) ratio and product exponential estimators are member of this class.

The bias and the $M S E$ of $t_{c}$ are respectively given by

$$
\begin{aligned}
& B\left(t_{c}\right)=V_{02} g_{2}+\bar{Y} V_{11} g_{3}+\bar{Y}^{2} V_{20} g_{4}, \\
& M S E\left(t_{c}\right)=\bar{Y}^{2} V_{20}+V_{02} g_{1}^{2}+2 \bar{Y} V_{11} g_{1} .
\end{aligned}
$$

where 


$$
g_{1}=\left.\frac{\partial g}{\partial u_{s t}}\right|_{\bar{y}_{s t}=\bar{Y}, u_{s t}=1}, g_{2}=\left.\frac{1}{2} \frac{\partial^{2} g}{\partial u_{s t}^{2}}\right|_{\bar{y}_{s t}=\bar{Y}, u_{s t}=1}, g_{3}=\left.\frac{1}{2} \frac{\partial^{2} g}{\partial \bar{y}_{s t} \partial u_{s t}}\right|_{\bar{y}_{s t}=\bar{Y}, u_{s t}=1}, g_{4}=\left.\frac{1}{2} \frac{\partial^{2} g}{\partial \bar{y}_{s t}^{2}}\right|_{\bar{y}_{s t}=\bar{Y}, u_{s t}=1} .
$$

By using the optimal value of $g_{1}^{*}=-\frac{\bar{Y} V_{11}}{V_{02}}$, the minimum $M S E$ of the estimators in the class $t_{c}$ is found as

$$
\operatorname{MSE}\left(t_{c}\right)_{\min }=\bar{Y}^{2} V_{20}\left[1-\frac{V_{11}^{2}}{V_{02} V_{20}}\right]
$$

which is also the MSE of combined regression type estimators and minimum MSE of Yadav et. al [8].

\section{Proposed Exponential Type Estimators}

Motivated by Koyuncu (2012) we propose the following estimator

$$
\bar{y}_{N}=\left[w_{1} \bar{y}_{s t}+w_{2}\left(\frac{\bar{x}_{s t}}{\bar{X}}\right)^{\gamma}\right] \exp \left[\frac{A_{s t}\left(\bar{X}-\bar{x}_{s t}\right)}{A_{s t}\left(\bar{X}+\bar{x}_{s t}\right)+2 B_{s t}}\right]
$$

Some new estimators, which are generated from (21) for different combinations of $A_{h}$, $B_{h}$ and $\gamma$ are given in Table 2. To obtain the MSE, we are applying the same procedure as follows:

$$
\begin{gathered}
\bar{y}_{N}=\left[w_{1} \bar{Y}\left(1+e_{0}\right)+w_{2}\left(1+e_{1}\right)^{\gamma}\right]\left\{1-\frac{\bar{X} A_{s t}}{2\left(\bar{X} A_{s t}+B_{s t}\right)} e_{1}+\frac{3}{8} \frac{\bar{X}^{2} A_{s t}^{2}}{\left(\bar{X} A_{s t}+B_{s t}\right)^{2}} e_{1}^{2}+\ldots\right\} \\
\bar{y}_{N}-\bar{Y}=\left\{\bar{Y}\left(w_{1}-1\right)+w_{1}\left(\bar{Y} e_{0}-\frac{1}{2} \psi \bar{Y} e_{1}-\frac{1}{2} \psi \bar{Y} e_{1} e_{0}+\frac{3}{8} \psi{ }^{2} \bar{Y} e_{1}^{2}\right)\right. \\
\left.+w_{2}\left(1+\gamma e_{1}+\frac{\gamma(\gamma-1)}{2} e_{1}^{2}-\frac{1}{2} \psi e_{1}-\frac{1}{2} \psi \gamma e_{1}^{2}+\frac{3}{8} \psi^{2} e_{1}^{2}\right)\right\}
\end{gathered}
$$

where $\psi=\frac{\bar{X} A_{s t}}{\left(\bar{X} A_{s t}+B_{s t}\right)}$.

Squaring both sides of (23) and taking expectation we have

$$
\begin{aligned}
\operatorname{MSE}\left(\bar{y}_{N}\right) & =E\left\{\bar{Y}^{2}+\bar{Y}^{2} w_{1}^{2}\left(1+e_{0}^{2}+\psi^{2} e_{1}^{2}-2 \psi e_{0} e_{1}\right)+w_{2}^{2}\left(1+\left(\gamma^{2}+\psi^{2}+\gamma(\gamma-1)-\psi \gamma-\gamma \psi\right) e_{1}^{2}\right)\right. \\
& +\bar{Y}^{2} w_{1}\left(\psi e_{0} e_{1}-2-\frac{3}{4} \psi^{2} e_{1}^{2}\right)+\bar{Y} w_{2}\left(\left(\psi \gamma-\frac{3}{4} \psi^{2}-\gamma(\gamma-1)\right) e_{1}^{2}-2\right) \\
& \left.+\bar{Y} w_{1} w_{2}\left(2+2(\gamma-\psi) e_{0} e_{1}+\left(2 \psi^{2}+\gamma(\gamma-1)-2 \gamma \psi\right) e_{1}^{2}\right)\right\}
\end{aligned}
$$

The MSE of the proposed estimator is given by

$$
\operatorname{MSE}\left(\bar{y}_{N}\right)=\left[\bar{Y}^{2} w_{1}^{2} H+w_{2}^{2} B+\bar{Y}^{2} w_{1} D+\bar{Y} w_{2} G+\bar{Y}^{2}+\bar{Y} w_{1} w_{2} F\right]
$$


where $H=\left(1+V_{20}+\psi^{2} V_{02}-2 \psi V_{11}\right), B=\left(1+\left(\gamma^{2}+\psi^{2}+\gamma(\gamma-1)-2 \gamma \psi\right) V_{02}\right)$,

$$
\begin{aligned}
& D=\left(\psi V_{11}-2-\frac{3}{4} \psi^{2} V_{02}\right), G=\left(\left(\psi \gamma-\frac{3}{4} \psi^{2}-\gamma(\gamma-1)\right) V_{02}-2\right) \\
& F=\left(2+2(\gamma-\psi) V_{11}+\left(2 \psi^{2}+\gamma(\gamma-1)-2 \gamma \psi\right) V_{02}\right)
\end{aligned}
$$

The optimum values of $w_{1}$ and $w_{2}$, obtained by minimizing (25) respectively, are given by

$$
w_{1(o p t)}=\frac{G F-2 D B}{\left(4 B H-F^{2}\right)} w_{2(o p t)}=\bar{Y} \frac{D F-2 G H}{\left(4 H B-F^{2}\right)}
$$

Substituting these values in (25) we get the minimum MSE of $\bar{y}_{N}$ as follows:

$$
\operatorname{MSE}\left(\bar{y}_{N}\right)=\bar{Y}^{2}\left[1-\frac{B D^{2}-D F G+H G^{2}}{\left(4 B H-F^{2}\right)}\right]
$$

Note that the optimum choice of the constants $w_{1}$ and $w_{2}$ involve unknown parameters. These quantities can be guessed quite accurately through pilot sample survey or sample data or experience gathered in due course of time (Koyuncu and Kadilar (2009, 2010). Secondly we consider following estimator

$$
\bar{y}_{M}=\left[w_{1}^{*} \bar{y}_{s t}+w_{2}^{*}\left(\frac{\bar{x}_{s t}}{\bar{X}}\right)^{\gamma}\right] \exp \left[\frac{A_{s t}^{*}-a_{s t}^{*}}{A_{s t}^{*}+a_{s t}^{*}+2 B_{s t}}\right]
$$

Some new estimators, which are generated from (28) for different combinations of $A_{h}$, $B_{h}$ and $\gamma$ are given in Table 2. Expressing (28) in terms of e's, we have

$$
\begin{aligned}
& \bar{y}_{M}=\left[w_{1}^{*} \bar{y}_{s t}+w_{2}^{*}\left(1+e_{1}\right)^{\gamma}\right]\left\{1-\frac{A_{s t}^{*}}{2\left(A_{s t}^{*}+B_{s t}\right)} e_{1}^{*}+\frac{3\left(A_{s t}^{*}\right)^{2}}{8\left(A_{s t}^{*}+B_{s t}\right)^{2}} e_{1}^{* 2}+\ldots\right\} \\
&\left(\bar{y}_{M}-\bar{Y}\right)=\left\{w_{1}^{*} \bar{Y}-\bar{Y}+w_{2}^{*}+w_{2}^{*} \frac{\gamma(\gamma-1)}{2} e_{1}^{2}-\frac{1}{2} \psi^{*} w_{1}^{*} \bar{Y} e_{0} e_{1}^{*}\right. \\
&\left.-\frac{1}{2} w_{2}^{*} \psi^{*} \gamma e_{1} e_{1}^{*}+\frac{3}{8} w_{1}^{*} \psi^{* 2} \bar{Y} e_{1}^{* 2}+\frac{3}{8} w_{2}^{*} \psi^{* 2} e_{1}^{* 2}\right\}
\end{aligned}
$$

where $\psi^{*}=\frac{A_{s t}^{*}}{\left(A_{s t}^{*}+B_{s t}\right)}$.

Squaring both sides of (30) and taking expectation we have

$$
\begin{aligned}
\operatorname{MSE} & \left(\bar{y}_{M}\right)=E\left\{\bar{Y}^{2}+w_{1} \bar{Y}^{2}\left(-2+\psi^{*} e_{0} e_{1}^{*}-\frac{3}{4} \psi^{* 2} e_{1}^{* 2}\right)+w_{1}^{2} \bar{Y}^{2}\left(1+e_{0}^{2}+\psi^{* 2} e_{1}^{* 2}-2 \psi^{*} e_{0} e_{1}^{*}\right)\right. \\
& +\bar{Y}_{w_{2}}\left(-2+\psi^{*} \gamma e_{1} e_{1}^{*}-\gamma(\gamma-1) e_{1}^{2}-\frac{3}{4} \psi^{* 2} e_{1}^{* 2}\right)+w_{2}^{2}\left(1+\left(\gamma^{2}+\gamma(\gamma-1)\right) e_{1}^{2}+\psi^{* 2} e_{1}^{* 2}-2 \gamma \psi e_{1} e_{1}^{*}\right) \\
& \left.+w_{1} w_{2} \bar{Y}\left(2-2 \gamma \psi^{*} e_{1} e_{1}^{*}+\gamma(\gamma-1) e_{1}^{2}-2 \psi^{*} e_{0} e_{1}^{*}+2 \psi^{* 2} e_{1}^{* 2}+2 \gamma e_{0} e_{1}\right)\right\}
\end{aligned}
$$


The MSE of the proposed estimator is given by

$$
\operatorname{MSE}\left(\bar{y}_{M}\right)=\left[\bar{Y}^{2}+w_{1}^{* 2} \bar{Y}^{2} H^{*}+w_{2}^{* 2} B^{*}+w_{1}^{*} \bar{Y}^{2} D^{*}+\bar{Y}_{2} w_{2}^{*} G^{*}+w_{1}^{*} w_{2}^{*} \bar{Y} F^{*}\right]
$$

where

$$
\begin{aligned}
& H^{*}=\left(1+V_{200}^{*}+\psi^{* 2} V_{020}^{*}-2 \psi^{*} V_{110}^{*}\right) \\
& B^{*}=\left(1+\left(\gamma^{2}+\gamma(\gamma-1)\right) V_{002}^{*}+\psi^{* 2} V_{020}^{*}-2 \gamma \psi^{*} V_{011}^{*}\right) \\
& D^{*}=\left(-2+\psi^{*} V_{110}^{*}-\frac{3}{4} \psi^{* 2} V_{020}^{*}\right) \\
& G^{*}=\left(-2+\psi^{*} \gamma V_{011}^{*}-\gamma(\gamma-1) V_{002}^{*}-\frac{3}{4} \psi^{* 2} V_{020}^{*}\right) \\
& F^{*}=\left(2-2 \gamma \psi^{*} V_{011}^{*}+\gamma(\gamma-1) V_{002}^{*}-2 \psi^{*} V_{110}^{*}+2 \psi^{* 2} V_{020}^{*}+2 \gamma V_{101}^{*}\right)
\end{aligned}
$$

The optimum values of $w_{1}$ and $w_{2}$ are given

$$
w_{1(o p t)}^{*}=\frac{G^{*} F^{*}-2 D^{*} B^{*}}{\left(4 B^{*} A^{*}-F^{* 2}\right)} \quad w_{2(o p t)}^{*}=\bar{Y} \frac{D^{*} F^{*}-2 G^{*} H^{*}}{\left(4 B^{*} A^{*}-F^{* 2}\right)}
$$

The minimum MSE of $\bar{y}_{M}$ at optimum values of $w_{1}^{*}$ and $w_{2}^{*}$, is given by

$$
\operatorname{MSE}\left(\bar{y}_{M}\right)=\bar{Y}^{2}\left[1-\frac{B^{*} D^{* 2}-D^{*} F^{*} G^{*}+H^{*} G^{* 2}}{\left(4 B^{*} H^{*}-F^{* 2}\right)}\right]
$$

\section{Numerical Example}

To analyze the performance of proposed estimator we use the data concerning the number of teachers as the study variable and the number of students as the auxiliary variable in both primary and secondary schools for 923 districts at 6 regions (as 1:Marmara 2:Agean 3:Mediterranean 4:Central Anatolia 5:Black Sea 6:East and Southeast Anatolia) in Turkey in 2007 (Source: Koyuncu and Kadilar (2009)). The summary statistics about the population is given in Table 1.

We have generated some new estimators using suitable values for $A_{h}, B_{h}$ and $\gamma$. The $M S E$ values of the $k_{1}, k_{2}, \bar{y}_{N}$ and $\bar{y}_{M}$ estimators have been obtained. These values are given in Table 2. When we examine Table 2, we observe that the proposed $\bar{y}_{M 4}$ estimator has the smallest. From Table 2 we can conclude that using suitable auxiliary information is very important otherwise we can get worst estimates in each class.

\section{Conclusion}

In this paper we have tried to generalize Yadav et al. (2014) estimators and corrected mean square error formula of Singh et al. (2008) and suggested two exponential type estimators in stratified random sampling. To see the performance of estimators we used a real data. We investigate the usage of suitable information of auxiliary variable. We have found that suggested exponential class of estimators is highly efficient than Yadav et al. (2014) and combined regression estimator. 


\section{References}

1. Diana G. A class of estimators of the population mean in stratified random sampling, Statistica, V.1, 1993, pp.59-66.

2. Singh H.P., Vishwakarma G.K. A family of estimators of population mean using auxiliary information in stratified sampling, Communications in Statistics-Theory and Methods, V.37, 2008, pp.1038-1050.

3. Koyuncu N., Kadilar C. Family of estimators of population mean using two auxiliary variables in stratified random sampling, Communications in Statistics: Theory and Methods, V.38, N.14, 2009, pp.2398-2417.

4. Koyuncu N., Kadilar C. On the family of estimators of population mean in stratified sampling, Pakistan Journal of Statistics, V.26, N.2, 2010, pp.427-443.

5. Koyuncu N., Kadilar C. On improvement in estimating population mean in stratified random sampling, Journal of Applied Statistics, V.37, N.6, 2010, pp.999-1013.

6. Tailor R., Chouhan S., Tailor R., Garg N. A ratio-cum-product estimator of population mean in stratified random sampling using two auxiliary variables, Statistica, V.LXXII. N.3, 2012, pp. 287-297.

7. Koyuncu N. Families of estimators for population mean using information on auxiliary attribute in stratified random sampling, Gazi University Journal of Science, V.26, N.2, 2013, pp.181-193.

8. Yadav R., Upadhyaya L.N., Singh H.P., Chatterjee S. Improved ratio and product exponential type estimators for finite population mean in stratified random sampling, Communications in Statistics: Theory and Methods, V.43, N.16, 2014, pp.3269-3285.

9. Singh R., Kumar M., Singh R. D., Chaudhary M.K. Exponential ratio type estimators in stratified random sampling, presented in International Symposium on Optimization and Statistics (I.S.O.S.) at AMU, Aligarh, India, 29-31 Dec., 2008.

10. Koyuncu N. Efficient estimators of population mean using auxiliary attributes, Applied Mathematics and Computation, V.218, 2012, pp.10900-10905. 
Table 1: Data Statistics

\begin{tabular}{|l|l|l|}
\hline$N_{1}=127$ & $N_{2}=117$ & $N_{3}=103$ \\
\hline$N_{4}=170$ & $N_{5}=205$ & $N_{6}=201$ \\
\hline$n_{1}=31$ & $n_{2}=21$ & $n_{3}=29$ \\
\hline$n_{4}=38$ & $n_{5}=22$ & $n_{6}=39$ \\
\hline$S_{y 1}=883.835$ & $S_{y 2}=644.922$ & $S_{y 3}=1033.467$ \\
\hline$S_{y 4}=810.585$ & $S_{y 5}=403.654$ & $S_{y 6}=711.723$ \\
\hline $\bar{Y}_{1}=703.74$ & $\bar{Y}_{2}=413$ & $\bar{Y}_{3}=573.17$ \\
\hline $\bar{Y}_{4}=424.66$ & $\bar{Y}_{5}=267.03$ & $\bar{Y}_{6}=393.84$ \\
\hline$S_{x 1}=30486.751$ & $S_{x 2}=15180.769$ & $S_{x 3}=27549.697$ \\
\hline$S_{x 4}=18218.931$ & $S_{x 5}=8497.776$ & $S_{x 6}=23094.141$ \\
\hline $\bar{X}_{1}=20804.59$ & $\bar{X}_{2}=9211.79$ & $\bar{X}_{3}=14309.30$ \\
\hline $\bar{X}_{4}=9478.85$ & $\bar{X}_{5}=5569.95$ & $\bar{X}_{6}=12997.59$ \\
\hline$S_{x y 1}=25237153.52$ & $S_{x y 2}=9747942.85$ & $S_{x y 3}=28294397.04$ \\
\hline$S_{x y 4}=14523885.53$ & $S_{x y 5}=3393591.75$ & $S_{x y 6}=15864573.97$ \\
\hline$\rho_{x y 1}=0.936$ & $\rho_{x y 2}=0.996$ & $\rho_{x y 3}=0.994$ \\
\hline$\rho_{x y 4}=0.983$ & $\rho_{x y 5}=0.989$ & $\rho_{x y 6}=0.965$ \\
\hline$\beta_{2}\left(x_{1}\right)=4.593$ & $\beta_{2}\left(x_{5}\right)=21.947$ & $\beta_{2}\left(x_{3}\right)=15.446$ \\
\hline$\beta_{2}\left(x_{4}\right)=10.162$ & & \\
\hline
\end{tabular}


Table 2: $\quad$ Some members of $k_{1}, k_{2}, \bar{y}_{N}$ and $\bar{y}_{M}$ and MSE values

\begin{tabular}{|c|c|c|c|c|}
\hline & $A_{h}$ & $B_{h}$ & $\gamma$ & MSE \\
\hline$t_{1}$ & $C_{x h}$ & & & 602.5943 \\
\hline$t_{2}$ & $\beta_{2 h}(x)$ & & & 603.8937 \\
\hline$t_{3}$ & $\beta_{2 h}(x)$ & $C_{x h}$ & & 688.4160 \\
\hline$t_{4}$ & $C_{x h}$ & $\beta_{2 h}(x)$ & & 589.5579 \\
\hline $\bar{y}_{N 1}$ & $\beta_{2 h}(x)$ & $C_{x h}$ & 0 & 53.1396 \\
\hline $\bar{y}_{N 2}$ & $\beta_{2 h}(x)$ & $C_{x h}$ & 1 & 556.4491 \\
\hline $\bar{y}_{N 3}$ & $\beta_{2 h}(x)$ & $C_{x h}$ & 2 & 342.4030 \\
\hline $\bar{y}_{N 4}$ & $C_{x h}$ & $\beta_{2 h}(x)$ & 0 & 52.1180 \\
\hline $\bar{y}_{N 5}$ & $C_{x h}$ & $\beta_{2 h}(x)$ & 1 & 557.3786 \\
\hline $\bar{y}_{N 6}$ & $C_{x h}$ & $\beta_{2 h}(x)$ & 2 & 342.5917 \\
\hline $\bar{y}_{N 7}$ & $\rho_{h(x y)}$ & $C_{x h}$ & 0 & 52.9641 \\
\hline $\bar{y}_{N 8}$ & $\rho_{h(x y)}$ & $C_{x h}$ & 1 & 556.6087 \\
\hline $\bar{y}_{N 9}$ & $\rho_{h(x y)}$ & $C_{x h}$ & 2 & 342.4354 \\
\hline $\bar{y}_{M 1}$ & $C_{x h}$ & $\beta_{2 h}(x)$ & 0 & 51.1939 \\
\hline $\bar{y}_{M 2}$ & $C_{x h}$ & $\beta_{2 h}(x)$ & 1 & 551.1354 \\
\hline $\bar{y}_{M 3}$ & $C_{x h}$ & $\beta_{2 h}(x)$ & 2 & 325.882 \\
\hline $\bar{y}_{M 4}$ & $\rho_{h(x y)}$ & $C_{x h}$ & 0 & 48.8562 \\
\hline $\bar{y}_{M 5}$ & $\rho_{h(x y)}$ & $C_{x h}$ & 1 & 505.5486 \\
\hline $\bar{y}_{M 6}$ & $\rho_{h(x y)}$ & $C_{x h}$ & 2 & 331.6634 \\
\hline$M S E_{\min }\left(t_{P C}^{(b)}\right)=M S E_{\min }\left(t_{R C}^{(a)}\right)$ & & & & 194.2832 \\
\hline
\end{tabular}




\section{Appendix}

$$
k_{2}=\bar{y}_{s t} \exp \left(\frac{A_{s t}^{*}-a_{s t}^{*}}{A_{s t}^{*}+a_{s t}^{*}+2 B_{s t}}\right)
$$

If we take $A_{h}$ as $\beta_{2 h}(x)$ and $B_{h}$ as $C_{x h}$ in $A_{s t}^{*}, a_{s t}^{*}$ and $B_{s t}$ respectively we can get $A_{s t}^{*}=\sum_{h=1}^{L} W_{h} \bar{X}_{h} \beta_{2 h}(x), a_{s t}^{*}=\sum_{h=1}^{L} W_{h} \bar{x}_{h} \beta_{2 h}(x), B_{s t}=\sum_{h=1}^{L} W_{h} C_{x h}$. Rewriting these values in (1) we get Singh et al (2008) estimator.

$$
t_{3}=\bar{y}_{s t} \exp \left(\frac{\bar{X}_{3}-\bar{x}_{3}}{\bar{X}_{3}+\bar{x}_{3}}\right)
$$

where $\bar{x}_{3}=\sum_{h=1}^{L} W_{h}\left(\bar{x}_{h} \beta_{2 h}(x)+C_{x h}\right)$

$$
\bar{X}_{3}=\sum_{h=1}^{L} W_{h}\left(\bar{X}_{h} \beta_{2 h}(x)+C_{x h}\right)
$$

To find the MSE formula let us define $e_{0}=\frac{\left(\bar{y}_{s t}-\bar{Y}\right)}{\bar{Y}}, e_{1}^{*}=\frac{a_{s t}^{*}-A_{s t}^{*}}{A_{s t}^{*}}=\frac{\sum_{h=1}^{L} W_{h} A_{h}\left(\bar{x}_{h}-\bar{X}_{h}\right)}{A_{s t}^{*}}$ such that $E\left(e_{0}\right)=E\left(e_{1}^{*}\right)=0, E\left(e_{0}^{2}\right)=\frac{\sum_{h=1}^{L} W_{h}^{2} \lambda_{h} S_{y h}^{2}}{\bar{Y}^{2}}, E\left(e_{1}^{* 2}\right)=\frac{\sum_{h=1}^{L} W_{h}^{2} A_{h}^{2} \lambda_{h} S_{x h}^{2}}{A_{s t}^{* 2}}$, $E\left(e_{0} e_{1}^{*}\right)=\frac{\sum_{h=1}^{L} W_{h}^{2} A_{h} \lambda_{h} S_{x y h}}{\bar{Y} A_{s t}^{*}}$

Expressing $k_{2}$ with e term, we have

$$
\begin{aligned}
& k_{2}=\bar{Y}\left(1+e_{0}\right) \exp \left(\frac{A_{s t}^{*}-A_{s t}^{*}\left(1+e_{1}^{*}\right)}{A_{s t}^{*}+A_{s t}^{*}\left(1+e_{1}^{*}\right)+2 B_{s t}}\right) \\
& k_{2}=\bar{Y}\left(1-\frac{1}{2\left(A_{s t}^{*}+B_{s t}\right)} A_{s t}^{*} e_{1}^{*}+\frac{3}{8} \frac{\left(A_{s t}^{*}\right)^{2}}{\left(A_{s t}^{*}+B_{s t}\right)^{2}} e_{1}^{* 2}+e_{0}-\frac{1}{2} A_{s t}^{*} e_{0} e_{1}^{*}+\frac{3}{8} \frac{\left(A_{s t}^{*}\right)^{2}}{\left(A_{s t}^{*}+B_{s t}\right)^{2}} e_{0} e_{1}^{* 2}\right) \\
& k_{2}-\bar{Y}=\bar{Y}\left(-\frac{A_{s t}^{*}}{2\left(A_{s t}^{*}+B_{s t}\right)} e_{1}^{*}+\frac{3}{8} \frac{\left(A_{s t}^{*}\right)^{2}}{\left(A_{s t}^{*}+B_{s t}\right)^{2}} e_{1}^{* 2}+e_{0}-\frac{1}{2} A_{s t}^{*} e_{0} e_{1}^{*}+\frac{3}{8} \frac{\left(A_{s t}^{*}\right)^{2}}{\left(A_{s t}^{*}+B_{s t}\right)^{2}} e_{0} e_{1}^{* 2}\right)
\end{aligned}
$$

Squaring both sides of (3) and neglecting the terms of e's having power greater than two, we have

$$
\left(k_{2}-\bar{Y}\right)^{2}=\bar{Y}^{2}\left(\frac{A_{s t}^{* 2}}{4\left(A_{s t}^{*}+B_{s t}\right)^{2}} e_{1}^{* 2}+e_{0}^{2}-\frac{A_{s t}^{*}}{\left(A_{s t}^{*}+B_{s t}\right)} e_{1}^{*} e_{0}\right)
$$


Taking expectation of both sides of (4) we get MSE of estimator $k_{2}$

$$
\begin{aligned}
M S E\left(k_{2}\right) & =\bar{Y}^{2}\left(\frac{1}{4} \frac{A_{s t}^{* 2}}{\left(A_{s t}^{*}+B_{s t}\right)^{2}} \frac{\sum_{h=1}^{L} W_{h}^{2} A_{h}^{2} \lambda_{h} S_{x h}^{2}}{A_{s t}^{* 2}}+\frac{\sum_{h=1}^{L} W_{h}^{2} \lambda_{h} S_{y h}^{2}}{\bar{Y}^{2}}-\frac{A_{s t}^{*}}{\left(A_{s t}^{*}+B_{s t}\right)} \frac{\sum_{h=1}^{L} W_{h}^{2} A_{h} \lambda_{h} S_{x y h}}{A_{s t}^{*} \bar{Y}}\right) \\
& =\sum_{h=1}^{L} W_{h}^{2} \lambda_{h}\left\{S_{y h}^{2}-\frac{\bar{Y} A_{h}}{\left(A_{s t}^{*}+B_{s t}\right)} S_{x y h}+\frac{1}{4} \frac{\bar{Y}^{2} A_{h}^{2}}{\left(A_{s t}^{*}+B_{s t}\right)^{2}} S_{x h}^{2}\right\} \\
& =\sum_{h=1}^{L} W_{h}^{2} \lambda_{h}\left\{S_{y h}^{2}-R_{k 2} S_{x y h}+\frac{1}{4} R_{k 2}^{2} S_{x h}^{2}\right\}
\end{aligned}
$$

where $R_{k 2}=\frac{\bar{Y} A_{h}}{\left(A_{s t}^{*}+B_{s t}\right)}$.

If we take $A_{h}$ as $\beta_{2 h}(x)$ and $B_{h}$ as $C_{x h}$ in $\operatorname{MSE}\left(k_{2}\right)$ we get corrected $\operatorname{MSE}\left(t_{3}\right)$ as

$$
\begin{aligned}
\operatorname{MSE}\left(t_{3}\right) & =\sum_{h=1}^{L} W_{h}^{2} \lambda_{h}\left\{S_{y h}^{2}-\frac{\bar{Y} \beta_{2 h}(x)}{\left(\sum_{h=1}^{L} W_{h} \bar{X}_{h} \beta_{2 h}(x)+\sum_{h=1}^{L} W_{h} C_{x h}\right)} S_{x y h}+\frac{1}{4} \frac{\bar{Y}^{2} \beta_{2 h}(x)^{2}}{\left(\sum_{h=1}^{L} W_{h} \bar{X}_{h} \beta_{2 h}(x)+\sum_{h=1}^{L} W_{h} C_{x h}\right)^{2}} S_{x h}^{2}\right\} \\
& =\sum_{h=1}^{L} W_{h}^{2} \lambda_{h}\left\{S_{y h}^{2}-R_{t 3} S_{x y h}+\frac{1}{4} R_{t 3}^{2} S_{x h}^{2}\right\}
\end{aligned}
$$

where $R_{t 3}=\frac{\bar{Y} \beta_{2 h}(x)}{\left(\sum_{h=1}^{L} W_{h}\left\{\bar{X}_{h} \beta_{2 h}(x)+C_{x h}\right\}\right)}$. 